\title{
Д.Ю. МАКСИмов
}

\section{Логика Н.А. Васильева и многозначные логики}

\author{
Максимов Дмитрий Юрьевич \\ Институт проблем управления им. В.А. Трапезникова РАН. \\ 117997, Российская Федерация, Москва, ул. Профсоюзная, д. 65. \\ E-mail: phoenixjhanjaa@yandex.ru
}

Рассматривается история понятия многозначности в логике с единых позиций как история отрицания. Основное внимание при этом уделяется логике Н.А. Васильева, ее связям с индийской логикой сйадвада, с логиками Я. Лукасевича, логикой Д.А. Бочвара. Выясняется многозначная природа логики Васильева, ее формальная модель в топосах. В топосах вводится новое понятие отрицания «в некотором смысле». Тогда множество типов суждения у Васильева оказывается множеством образующих дистрибутивной решетки истинностных значений топоса, которые переводятся друг в друга отрицанием «в соответствующем смысле», а васильевское понимание отрицания соответствует решеточному отрицанию (псевдодополнению). Обсуждается понимание Васильевым закона исключенного $n$-го и паранепротиворечивости: Н.А. Васильев рассматривал свой закон исключенного $n$-го как дизъюнкцию образующих, как дизъюнкцию разных способов построения отрицательного суждения «в некотором смысле», а закон непротиворечия как конъюнкцию утверждения и его отрицания «в некотором смысле». Указаны условия для моделирования этих законов в категориях.

Ключевые слова: Логика Васильева, многозначная логика, категорная семантика.

\section{1. Введение}

В многозначных логиках, в отличие от классической двузначной логики, истинность высказывания может отличаться от true и false и принимать значения во множествах, имеющих больше двух элементов. Это значит, что высказывания могут быть не только истинными или ложными. Есть несколько следующих основных источников возникновения таких логических систем:

- Во-первых, это возможная зависимость истинности высказывания от контекста, в котором оно употребляется, а также гносеологические идеи скептицизма.

- Во-вторых, это неполнота информации, которая требуется для однозначной оценки высказывания как истинного или ложного.

- В-третьих, это временная неопределенность истинности высказываний о будущих событиях.

- В-четвертых, это нечеткость понятий, относительно которых делается высказывание. 
В основном из первого источника возникла многозначность в системах логики Древней Индии (§ 2). Анализ понятия частного высказывания в аристотелевской логике выявил наличие в таких высказываниях неполноты информации и привел Н.А. Васильева в начале ХХ века к логике, которая очень похожа на индийские системы, но также содержит оригинальную интерпретацию отрицания (§3).

Попытки оценить истинность высказываний о будущих событиях имеют богатую историю. Еще Аристотель, не вводя третьего истинностного значения, не распространял действия принципа двузначности (согласно которому любое высказывание или только истинно, или только ложно) на будущие события. В противном случае не было бы ни свободы выбора, ни случайных событий. В средние века У. Оккам, по сути, вводит третье истинностное значение для будущих событий, которое он интерпретирует как «неопределенно», для согласования теологической концепции Божественного предвидения со свободной волей человека. В первой половине XX века Я. Лукасевич, из сходных с Аристотелем соображений, создает сначала трехзначную логику, а затем и многозначные системы с линейной шкалой истинностных значений (§ 4). Впоследствии, на основе трехзначной логики Лукасевича и из иных соображений появилось много других вариантов, что связано со значительной свободой построения истинностных таблиц для логических связок и отсутствием содержательной семантики для такой логики.

Наконец, из идей нечеткости понятий (например, «молодой», «куча» и др.) и приближенных рассуждений в середине XX века появилась нечеткая логика Л. Заде с такой же линейной шкалой истинностных значений, как у Лукасевича (§ 5). Впоследствии появились нечеткие логики с нелинейными шкалами.

В данной работе мы рассмотрим понятие многозначности во всех этих системах с единых позиций, как историю понятия отрицания. Основное внимание при этом будет уделено логике Н.А. Васильева, ее связям в древнеиндийской логикой, логикой Лукасевича и др. Выясняется многозначная природа логики Васильева, предлагается ее формальная модель в топосах. Обсуждается понимание Васильевым закона исключенного $n$-го и паранепротиворечивости.

Определение отрицания связано со структурой шкалы истинностных значений, на которой отрицание определяется. Поэтому вторая линия развития идет по используемым шкалам: у Лукасевича и в родственных (по шкале) логиках - линейная решетка, в 4-значной модальной логике Лукасевича и в логике Белнапа - простейшая дистрибутив- 
ная решетка, в логике Васильева - это общая дистрибутивная решетка (или даже недистрибутивная в обобщении в топосах), в нечеткозначной логике - дальнейшее обобщение понятия решетки.

\section{2. Многозначность в логике Древней Индии ${ }^{1}$}

Первые следы скептицизма, выражающие наличие гносеологических ситуаций с неустранимым сомнением, встречаются уже в «Ригведе». И это приводит к отрицанию принципа двузначности и признанию возможности одновременного существования и не-существования: « Тогда не было ни сущего, ни не-сущего. .. » [5]. Впоследствии адживики пришли к выводу, что также возможна ситуация, когда нечто «и есть, и не есть», что к ряду предметов можно применять двойственные определения: «и мир, и не-мир», «и душа, и не-душа» и проч. [21]. Таким образом, к VI-IV вв. до н.э. в Индии существовало четыре способа описания реальности: о любом предмете можно сказать, что он есть, или не есть, или и есть и не есть, или ни есть ни не есть. Такая схема получила название «чатушкуотика», т.е. «имеющая четыре вершины». Эту концепцию, как и возникшие на ее основе доктрины аджнянавада и сйадвада, использовали все направления индийской философии, но наибольшее распространение она получила у буддистов. При этом буддизм, отвергая возможность выразить в словах, с помощью логических рассуждений, объективную реальность, использовал «чатушкуотику» в чисто пропедевтических целях.

Если буддизм отвергал всякую возможность выразить истину в слове, то джайны только сомневались в такой возможности и, в частности, выразить истину вариантами «чатушкуотики». Поэтому они дополнили четырехвершинную схему пятой вершиной: «Я не говорю этого. Я не говорю так. Я не говорю иначе. Я не говорю нет. Я не говорю не нет» [17]. Эта концепция получила название аджнянавада и может быть проинтерпретирована следующим образом.

1) Неверно, что объект есть. 2) Неверно, что он не есть. 3) Неверно, что он есть и не есть. 4) Неверно, что он ни есть, ни не есть. 5) Неверно, что 1)-4) истинны. Действительно, «так» значит не «иначе»; «нет» означает ни «так», ни «иначе»; «не нет» отрицает все эти три возможности. Наконец, «Я не говорю этого» отрицает отрицания всех других четырех суждений. При этом термин «неверно» не означает, что отрицающие друг друга пять высказываний являются взаимоисключающими.

\footnotetext{
${ }^{1}$ На основании [17].
} 
Таким образом, слово «аджнянавада» обозначает некий скептицизм, когда сторонник этой доктрины не знает, знает ли он или нет что-либо о предмете. Впоследствии джайны разработали более сложную доктрину сйадвада, в которой о любом объекте можно высказаться семью способами: 1) Объект некоторым образом существует; 2) Некоторым образом не существует; 3) Некоторым образом и существует и не существует; 4) Некоторым образом непредикативен; 4) Некоторым образом и существует и непредикативен; 6) Некоторым образом и не существует и непредикативен; 7) Некоторым образом и существует, и не существует, и непредикативен [17]. Опять-таки, как и в аджнянаваде, все эти семь высказываний не являются взаимоисключающими. Термин «сйад» («некоторым образом») указывает на зависимость истинности высказывания от контекста, на ограниченность и конкретность утверждения, на истинность относительно определенных предпосылок.

Так, например, в ситуации 1), когда говорится, что кувшин некоторым образом существует, имеется в виду некоторый конкретный, например этот глиняный, кувшин. Тогда, если говорить, например, о серебряном кувшине, то высказывание 1) будет ложным, а истинным возможно будет высказывание 2). В свою очередь высказывание 2) трактует небытие предмета как бытие некоторого другого предмета. Иначе говоря, если кувшин некоторым образом не есть, то это означает, что он некоторым образом не кувшин, а кусок обожженной глины, или выполняет другие, не свойственные кувшину функции или просто другой кувшин, не тот, который имелся в виду.

Высказывание 3) можно толковать и как совпадение в разных степенях бытия и небытия, и как существование с одной точки зрения и несуществование с другой. Например, рассматривая одну часть предмета с точки зрения его собственных свойств, а другую - с точки зрения чуждых ему свойств. В отличие от этого, высказывание 4) относится ко всему предмету в целом и утверждает его некоторым образом неописуемость (например, демонов) или отсутствие ответа на данный вопрос. Например, цвет предмета может зависеть от освещения, и каков он в действительности, непонятно. То есть в данном случае любое словесное описание неполно и зависит от точки зрения субъекта.

Истинность остальных высказываний рассматривается также с точки зрения соотношения собственных и чуждых свойств разных частей объекта и, кроме того, с учетом невозможности описать предмет вообще. 
Следует подчеркнуть, что все упомянутые логические системы имеют дело с объектным рядом, с конкретными вещами, но не с идеями и понятиями [13]. Истинность суждений о предметах находится «в зависимости не просто от склонности судящего рассудка, но от относительного характера самой многогранной реальности» [18]. В начале XX века Н.А. Васильев тоже пришел к выводу о необходимости разделять в логике уровень понятий и уровень фактов, но, в отличие от индийских логиков, у него принцип двузначности относится к уровню фактов, о которых можно делать однозначные утверждения, а вот о понятиях можно высказываться многими способами и для них должен выполняться закон исключенного четвертого. Впоследствии Васильев обобщает свои взгляды и на уровень фактов, но в воображаемом мире, и приходит в этом случае к закону исключенного $n$-го.

\section{3. Логика Н.А. Васильева}

В предыдущем разделе мы видели, что в индийских логических системах к идее многозначности приходят, в основном, из варьирования гносеологических принципов, из идей скептицизма. Н.А. Васильев выделяет в логике два слоя. Один слой относится к познающему субъекту - это, в его терминологии, металогика. Это законы, которые касаются суждения в целом и носят гносеологический характер. Один из них - закон исключенного третьего: «всякое суждение или истинно или ложно». И эти законы металогики, гносеологические принципы Васильев не варьирует: «мы предполагаем неизменность познающего субъекта и его рациональных функций - способности суждения и вывода» [2]. Но Васильев варьирует принципы, зависящие от познаваемых объектов. В этом слое он также выделяет два логических уровня. Один уровень относится к суждениям о фактах, о результатах наблюдения, опыта. K другому уровню относятся суждения о понятиях, о законах. Такие суждения передают не существования, а закон, связь между существованиями. Для разных систем объектов, разных миров, законы онтологического, эмпирического уровня могут различаться. Васильев рассматривает два таких закона - закон противоречия в формулировке Канта: «ни одной вещи не может принадлежать предикат, противоречащий ей», - и закон исключенного третьего в его онтологической формулировке [2]. Именно эти законы, относящиеся к вещам, Васильев и предлагает модифицировать. 


\section{1. О частных суждениях и законе исключенного четвертого}

Свою первую статью [3] Н.А. Васильев посвящает трактовке частных суждений в силлогистике. В силлогистике суждения делятся на общие и частные, что дает четыре формы: общеутвердительное $A$, общеотрицательное $E$, частноутвердительное $I$, частноотрицательное $O$. Частное суждение выражается формулой: «некоторые $S$ суть (не суть) $P$ ». Как указывает Васильев, знак частности может иметь два смысла: 1) некоторые, а может быть и все, по крайней мере некоторые; 2) некоторые, но не все, только некоторые. Анализируя первый вариант, Васильев приходит к выводу, что такой смысл могут иметь только предложения, но не суждения. Это проблематическое, неопределенное высказывание. Но наука не может иметь дело с неопределенными высказываниями, поэтому, делает вывод Васильев, слово «некоторые» в частных суждениях может иметь только смысл «не все». Поскольку «некоторые $S$ суть $P$ », а остальные «не суть $P »$, то оба частных высказывания $I$ и $O$ представляют одно общее высказывание $M:$ «все $S$ или суть $P$, или не суть $P$ ». Такое высказывание Васильев называет индифферентным.

Для этого высказывания Васильев предлагает две формы трактовки. Одна - дизъюнктивная, которая предполагает, что весь объем $S$ распределен по признакам $P, Q, R \ldots$ - «некоторые $S$ суть $P »$, «некоторые $S$ суть $Q »$, «некоторые $S$ суть $R$ ». Другая форма - акцидентальная, которая предполагает, что признак $P$ не обязателен для $S$, случаен, возможен, что предикат $P$ совместим с природой $S$. То есть акцидентальное суждение выражает определенное отношение между понятиями, их частичное совпадение. Это суждение не проблематическое, а утверждает некое правило. Напротив того, проблематическое относится всегда к фактам, а не к понятиям, выражает предположение о фактическом отношении. Такими частными суждениями о факте могут быть единичные, групповые, числовые или неопределенно-числовые («несколько $S$ есть $P \gg)$ суждения и именно для них, доказывает Васильев, верна традиционная силлогистика Аристотеля с обычным квадратом противоположностей и законом исключенного третьего. Это неудивительно, поскольку, как мы видели в $\S 2$, античное (индийское) мышление имело дело с отношениями между фактами, а не между понятиями. И греческая логика в этом пункте не отличается от индийской.

Если же иметь дело с понятиями, то для частных высказываний в трактовке Васильева вместо квадрата противоположностей получается треугольник противоположностей: «любая пара суждений $A, E, M$ не 
может быть одновременно истинной, но может быть одновременно ложной». Отсюда следует закон исключенного четвертого для понятий, т.е. что истинно только одно из трех утверждений: $A$ или $E$ или $M$.

Таким образом, для любого понятия и всякого его предиката может быть три возможности:

- Либо понятию присущ данный предикат;

- Либо ему присущ противоречащий предикат;

- Либо ему присущ и тот, и другой, т.е. оба предиката совместимы с данным понятием (как, например, с понятием «человек» совместимы предикаты «брюнет» и «не брюнет»).

Можно то же выразить идеей модальности: каждый предикат или необходим, или невозможен, или возможен для данного понятия.

В этой своей первой статье Н.А. Васильев пока не рассматривает закон противоречия. Но впоследствии он развивает свою идею о возможной совместимости признаков и приходит к понятию неклассического отрицания, которое вообще не основано на понятии совместимости и получает в этом случае закон исключенного $n$-го и нарушение закона противоречия.

\section{2. Закон паранепротиворечия и понятие отрицания}

В своей второй статье [4] Н.А. Васильев переходит к логике, которая могла бы иметь место для предметов и субъектов в некоем воображаемом мире. Отталкиваясь от идей Лобачевского, построившего неевклидову геометрию, отказавшись от 5-го постулата Евклида, Васильев также предлагает отказаться от одного из законов логики Аристотеля. И свою воображаемую логику Васильев строит без закона противоречия. Закон противоречия, согласно Васильеву, выражает несовместимость утверждения и отрицания. Но отрицание - это, по определению, есть то, что не совместимо с утверждением. Поэтому, заключает Васильев, строить логику без закона противоречия значит строить логику с отрицанием, не основанном на несовместимости.

Отрицательное суждение имеет два аспекта. Первый - формальный: отрицательное суждение высказывает ложность утвердительного. Второй - материальный: отрицательное суждение основывается на несовместимости предикатов. Этот аспект указывает, на каком основании мы приходим к истинности отрицательных суждений. Изменив такое основание, можно прийти к неклассическому отрицанию.

Н.А. Васильев указывает, что утвердительное суждение основывается на непосредственном восприятии и ощущении, а отрицательное - 
это всегда вывод. Мы не воспринимаем «не белое», а делаем вывод, что предмет не белый на том основании, что он какого-либо другого цвета. Но в воображаемом мире отрицательные суждения могут быть такими же непосредственными, как и положительные, где возможно непосредственное восприятие отрицательных ощущений и сам опыт в этом убеждает без всякого вывода. Поэтому в таком мире могут в одном предмете возникнуть основания и для утвердительного и для отрицательного суждений. Суждение, которое выражает присутствие в объекте оснований и для положительного и для отрицательного суждения, Васильев называет индифферентным.

Мы уже встречались в предыдущем разделе с индифферентными суждениями, но там они соотносились с частными суждениями, с высказыванием о классе и означали, что некоторый подкласс обладает данным предикатом, а другие подклассы не обладают им. Это были суждения о понятиях, выражающие некоторую закономерность, отношение между ними. Здесь же речь идет о суждениях единичных, о суждениях об отдельных фактах, которые выражают эмпирическую реальность мира, не совпадающего с нашим.

Далее Васильев строит силлогистику по той же схеме, что и в первой статье (см. § 3.1). Общие суждения, т.е. суждения о классе $S$, о понятии могут быть трех видов: 1) общеутвердительное суждение - все единичные $S$ обладают предикатом $P$, все $S$ суть $P ; 2)$ общеотрицательное суждение - все $S$ не суть $P ; 3)$ индифферентное суждение - все $S$ суть и не суть $P$. Кроме общих суждений могут быть еще частные, когда только некоторые $S$ обладают данным предикатом. Васильев опять называет их акцидентальными и выделяет четыре вида таких суждений: 4) некоторые $S$ суть $P$, а все остальные не суть $P ; 5)$ некоторые $S$ суть $P$, а все остальные суть и не суть $P ; 6$ ) некоторые $S$ не суть $P$, а все остальные суть и не суть $P ; 7)$ некоторые $S$ суть $P$, некоторые $S$ не суть $P$, а все остальные суть и не суть $P$.

Мы видим, что Васильев в своих предположениях получает столько же форм (а именно семь), сколько их выделяют аджнянавадины в концепции сйадвада (см. § 2). Причем, все эти формы практически дословно совпадают, если заменить термин «непредикативен» сйадвады на индифферентное суждение Васильева. Но получены они из разных гносеологических предпосылок и относятся к разным уровням: у Васильева - к уровню понятий, к классам, а у аджнянавадинов - к уровню фактов, к единичным объектам. Правда, схема рассуждений в аджнянаваде схожа со схемой Васильева: в предмете выделяются раз- 
ные части, к которым можно относить разные суждения, и отрицание признака вещи понимается как наличие признака другой вещи. Можно представить, что скептицизм аджнянавады связан с предположением того, что реальные объекты «живут» в воображаемом мире Васильева и мы имеем дело с его проекцией на наш мир.

Н.А. Васильев в своих рассуждениях о воображаемой логике исходил из возможности в воображаемом мире непосредственного восприятия, ощущения как наличия признака, так и его отсутствия. В таком мире есть три качественно различных суждения - утвердительное, отрицательное и индифферентное и, поэтому, имеет место закон исключенного четвертого. Но можно также мыслить миры с $n$ качественно различными суждениями, из которых мы можем иметь дело только с утверждением и отрицанием. В таком мире уже будет иметь место закон исключенного $n+1$-го, который утверждает наличие $n$ форм суждений и исключает $n+1$ форму.

Интересно отметить, что Н.А. Васильев не вводит иерархию форм суждений по степени истинности. Поэтому у него нет проблемы с выбором значения для отрицания индифферентного суждения, как у Лукасевича (см. § 4). Он вводит дополнительный тип неопределенного суждения, как колебания между двумя другими возможностями, что аналогично аристотелевскому пониманию частного высказывания: «некоторые, а может быть и все» (см. § 3.1). Такие неопределенные суждения берутся в качестве отрицания каждой из трех форм суждений и связывают воображаемую логику с логикой Аристотеля. Таким образом, по существу, Васильев предложил в качестве множества истинностных значений дистрибутивную решетку с тремя образующими (см. след. разд.). В этой шкале логические связки определяются как решеточные операции, т.е. отрицание определяется как псевдодополнение, дизъюнкция и конъюнкция - как объединение и пересечение соответственно, а импликация $Z=A \rightarrow B$ как наибольший элемент, который имеет пересечение с $A$ такое же, как $B$.

\section{3. Формальная модель идей Н.А. Васильева}

К сожалению, Н.А. Васильев не успел перевести свою систему на язык символической логики, а сама система содержит большое разнообразие идей, порой неясно выраженных. Поэтому существуют многообразные формальные модели его взглядов. При этом в немногочисленных известных работах логику Н.А. Васильева рассматривают, в основном, не как многозначную, а как предшественницу паранепротиворечивых или модальных логик и с этих позиций и пытаются моделировать [20], [16]. 
Так, А. Арруда исходит из того, что в логике Васильева два уровня логический и металогический, поэтому она вводит два типа отрицания: для одного выполняется закон противоречия, для другого нет, что близко идеям Д.А. Бочвара [1] (см. § 4). Причем, не обязательно вводить для этого два символа - можно ввести два типа пропозициональных букв с разными правилами для них. В варианте с двумя символами отрицания вводятся также две конъюнкции, что приводит к формально более близким к взглядам Васильева выводам: для неклассического отрицания $\sim$ закон исключенного третьего $X \vee \sim X$ не выполняется, но выполняется закон исключенного четвертого $X \vee \sim X \vee(X \cdot \sim X)$, где - - неклассическая конъюнкция.

В.А. Смирнов рассматривает расширение классической логики, добавляя к ее структурным правилам силлогистические в целях аксиоматизации в силлогистике взглядов Васильева. Он вводит четыре модальных оператора - необходимости, случайности, возможности и детерминированности, что в совокупности делает аксиоматику такой силлогистики очень громоздкой.

В этом же направлении интерпретируется логика Васильева в [9]. Т.П. Костюк и В.И. Маркин построили силлогистическое исчисление, аксиоматизирующее класс общезначимых в этой семантике формул, доказали его непротиворечивость и полноту.

Однако существуют и многозначные интерпретации логики Васильева, например, перевод логики Васильева в язык многозначной логики предикатов [15]. В.И. Маркин доказал, что данный перевод погружает воображаемую логику в кванторную трехзначную логику, т.е. формула является законом воображаемой логики тогда и только тогда, когда ее перевод доказуем в трехзначной логике предикатов.

Основываясь на указании Н.А. Васильева о возможности двух типов отрицательных суждений - абсолютном и слабом, - в [6] Д.В. Зайцев и В.И. Маркин предложили интенсиональную семантику логики Васильева и соответствующее аксиоматическое исчисление.

Многозначная категорная семантика для идей Н.А. Васильева предложена в работе [25] (первые определения в [10]). В этой работе предложена модель взглядов Васильева в рамках аксиом пропозициональной части интуиционистской логики с добавлением аксиомы «исключенного $n$-го», что имеет место в определнном классе топосов (см. ниже). Автор исходит из наблюдения Васильева, что высказывание «некоторые $S$ есть $P$ » соответствует третьему способу сравнения понятий $S$ и $P$ и определяется пересечением $S$ и $P$. В современной ма- 
тематической логике этому высказыванию больше всего соответствует понятие степени эквивалентности $S$ и $P-[S \equiv P]$, в соответствии с которым $S$ эквивалентно $P$ там, где они пересекаются, или там, где оба не определены (рис. 1а).

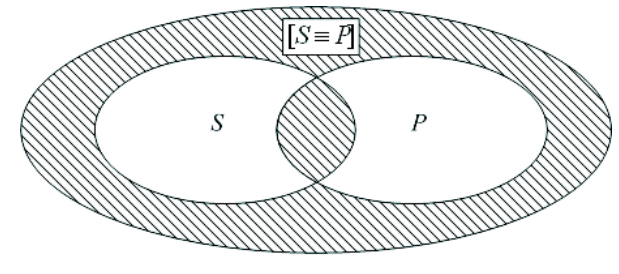

$$
[S \equiv P]=S \cup P \Rightarrow S \sim P
$$

a

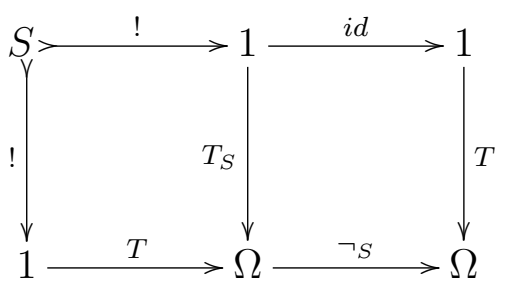

b

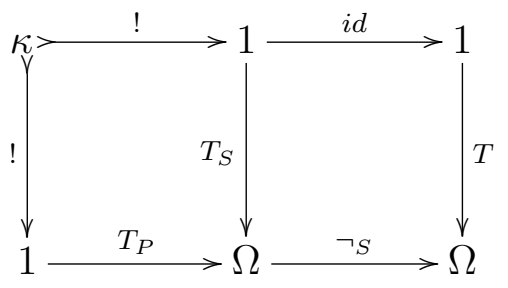

C

Рис. 1

Автор вводит понятие отрицания «в смысле $S »-\neg S$, как характеристическую стрелку истинностного значения $T_{S}$, которое само является характером подобъекта $S$ конечного объекта 1 в некотором топоce (рис. 1b, где $\Omega$ - объект истинностных значений этого топоса; для классической двузначной логики это просто множество $\{0,1\})$. Доказывается, что степень эквивалентности $S$ и $P$ совпадает с отрицанием 
$P$ «в смысле $S »($ или $S$ «в смысле $P$ »), т.е. это особый тип отрицания, который зависит от степени пересечения денотатов: на рис.1с для характеристической стрелки $T_{\kappa}$ объекта $\kappa=[S \equiv P]$ имеем $T_{\kappa}=\neg_{S} T_{P}$.

Выяснено, что в специальном классе топосов выполняется закон «исключенного $n$-го», но в другом понимании, нежели у Васильева (см. ниже): $\bigvee_{\mu \in S u b(1)} \neg_{S} P$, где дизъюнкция берется по всем элементам решетки истинностных значений. Такими специальными топосами являются, в частности, слабо экстенсиональные топосы, в которых неравные стрелки могут быть различимы частичным элементом. Такие топосы могут быть классическими и неклассическими. В последнем случае закон исключенного третьего в них не выполняется. Но могут быть и сильно неклассические топосы, в которых не выполняется и закон исключенного $n$-го. Таким образом в топосах могут моделироваться классическая логика, вариант логики Васильева или интуиционистская логика в зависимости от типа топоса. Такая категорная модель согласуется с представлениями Васильева о $n$-1 качественно различных типах отрицания и позволяет уточнить, что понимается под отрицанием у Васильева и в индийской концепции сйадвада.

Действительно, в своей логике Васильев берет в качестве отрицания каждого из трех типов высказывания высказывание, колеблющееся между двумя другими типами. Такое отрицание может соответствовать понятию отрицания в дистрибутивной решетке, например, в решетке истинностных значений некоторого топоса. Так, на рис. 2 изображена решетка истинностных значений некоторого классического топоса, состоящая из восьми элементов. Этот рисунок представляет верхнюю полурешетку с тремя образующими $\sim P, P, I$ (достроенную пунктиром до решетки) семи способов высказывания в воображаемой логике Васильева (и, как мы видели, в сйадваде).

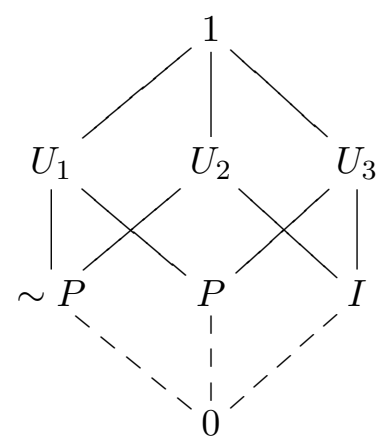

Рис. 2 
$I$ обозначает индифферентное высказывание «все $S$ суть и не суть $P$ », $P$ - высказывание «все $S$ суть $P$ » $\sim P$ - «все $S$ не суть $P », U_{1}-$ решеточное отрицание $I-\neg I$ соответствует колебанию между $\sim P$ и $P$ и может иметь интерпретацию Васильева 4$)$ : «некоторые $S$ суть $P$, а все остальные не суть $P \gg(\S 3.2)$. Так же интерпретируются остальные элементы полурешетки. В таком случае отрицание $I$, например, «в смысле $P \gg-\neg P I$ будет третьей образующей: $\neg P I=\neg U_{3}=\sim P$.

Таким образом, Н.А. Васильев различал отрицательное суждение $\sim P$ и отрицание суждения $P$, так что его операцию отрицания можно трактовать как решеточное отрицание (псевдодополнение), а формы высказывания о понятии («все $S$ суть $P$ », «все $S$ не суть $P$ », «все $S$ суть и не суть $P \gg)$ как образующие этой решетки, которые, как мы видим, переводятся друг в друга не обычным отрицанием, как у Аристотеля, а отрицанием «в смысле $P$ » (или «в смысле $\sim P$ », или «в смысле $I »)$.

Н.А. Васильев считал, что в законе «исключенного четвертого» истинно всегда одно утверждение из трех возможных - утвердительное, отрицательное или индифферентное, а два других ложны. Но если в решетке на рис. 2 одну из образующих принять за true, то решетка перестроится так, что две других образующих примут значение false [14]. Поэтому можно представить закон «исключенного четвертого» в современной форме - как дизъюнкцию образующих, которая всегда истинна. Таким образом, можно рассматривать закон исключенного $n$-го как дизъюнкцию разных способов построения отрицательного суждения «в некотором смысле». Поскольку дизъюнкция образующих все равно дает наибольший элемент решетки, то такое объединение эквивалентно объединению всех элементов решетки, всех истинностных значений $\bigvee \neg_{S} P$. Но решетка на рис. 2 булева, поэтому несмотря на то что $\mu \in \operatorname{Sub}(1)$

в топосе с такой решеткой все равно верен закон «исключенного 4-го» и в васильевском понимании, и в форме $\bigvee_{\mu \in \operatorname{Sub}(1)} \neg_{S} P$, в топосах с такой решеткой истинностных значений верен обычный закон исключенного третьего: $X \vee \neg X$, где $\neg-$ решеточное отрицание (псевдодополнение). Таким образом, логика в топосах с такой решеткой хоть и многозначная, но на самом деле классическая. Чтобы логика была неклассической, но с законом «исключенного $n$-го», топос должен быть устроен определенным образом [25], например, быть слабо экстенсиональным. В таких топосах решетка истинностных значений небулева, а это значит, что образующие имеют нетривиальные пересечения. То есть для того, чтобы воображаемая логика была неклассической, некоторые из 
качественно различных отрицаний все-таки должны иметь пересекающиеся денотаты, как предполагал Н.А. Васильев в своей первой работе [3]. В этом случае такая логика будет паранепротиворечивой по Васильеву - конъюнкция утвердительного суждения и отрицательного суждения не ложна, т.е. в ней не выполняется закон непротиворечия в форме $P \wedge \sim P \rightarrow 0$, поскольку $P$ может иметь ненулевое пересечение с $\sim P=\neg_{I} P$. Но для того чтобы не выполнялся обычный закон непротиворечия $P \wedge \neg P \rightarrow 0$, следует ввести неклассическую конъюнкцию и неклассическое отрицание, как это сделано в символическом виде в [20] (см. также начало этого параграфа) или для случая линейной логики проинтерпретировано в [25] в моноидальных категориях.

\section{4. Логики Я. Лукасевича и родственные им}

Я. Лукасевич пришел к созданию трехзначной логики в 20-х годах XX века, исследуя аристотелевскую проблему истинностного статуса будущих случайных событий [12]. Проблема состоит в том, что мы не можем сказать, истинно ли, например, утверждение: «Ян завтра в полдень будет дома», поскольку мы не знаем этого достоверно. Но точно так же мы не можем сказать, что это утверждение ложно, потому что для этого сегодня тоже нет оснований. Вместе с тем, дизъюнкция «Ян завтра в полдень будет дома или Ян завтра в полдень не будет дома», несомненно, истинна. Из этого Лукасевич делает вывод, что неверен принцип бивалентности, который состоит в том, что каждое высказывание либо истинно, либо ложно, и могут быть высказывания, которые ни истинны, ни ложны. Такие высказывания Лукасевич называет «безразличными». Интересно сравнить такие высказывания с индифферентными у Н.А. Васильева. Васильев предполагает, что единичные индифферентные высказывания относятся к материальному уровню и содержат в себе основания и для положительного и для отрицательного суждения. Лукасевич же предполагает, что таких оснований нет ни для одного, ни для другого. Тем самым, эти два логика в совокупности реализовали схему чатушкуотики (§ 2). Но у Васильева истинность суждений не упорядочена, а Лукасевич предлагает линейно-упорядоченную шкалу истинностных значений.

Васильев работал в рамках силлогистики и не успел представить свою систему даже в символическом виде. Лукасевич с самого начала разрабатывал математическую теорию трехзначной, а впоследствии и многозначной логики, что, однако, привело к несоответствию формальной теории исходным философским выводам. Дело в том, что простое 
добавление третьего истинностного значения требует переопределения логических связок, а сделать это можно, формально, разными способами, что приводит к разным трехзначным логикам, ни одна из которых, тем не менее, не соответствует исходным смысловым посылкам Лукасевича в полной мере. Это связано, возможно, с тем, что простое добавление третьего истинностного значения может привести только к линейной шкале истинностных значений, в то время как добавление индифферентного суждения Васильевым приводит, как мы видели в предыдущем разделе, к нелинейной решетке истинностных значений с тремя образующими.

В своей первой системе [23], в 1920 г., Я. Лукасевич берет в качестве исходных связок отрицание $\sim$ и импликацию $\rightarrow$, для которых оставляет классические значения тогда, когда аргументы принимают значения в множестве $\{0,1\}$. В остальных случаях Лукасевич доопределяет эти операции следующим образом: $(1 \rightarrow 1 / 2)=(1 / 2 \rightarrow 0)=1 / 2 ; \quad(0 \rightarrow 1 / 2)=$ $(1 / 2 \rightarrow 1 / 2)=(1 / 2 \rightarrow 1)=1 ; \sim 1 / 2=1 / 2$.

Другие логические связки определяются через исходные:

$P \vee Q=(P \rightarrow Q) \rightarrow Q ; \quad P \wedge Q=\sim(\sim P \vee \sim Q)$

Легко проверить, что в логике Лукасевича не выполняются:

- Закон сокращения $(P \rightarrow(P \rightarrow Q)) \rightarrow(P \rightarrow Q)$;

- Закон исключенного третьего $P \vee \sim P$;

- Закон непротиворечия $\sim(P \wedge \sim P)$.

Все эти формулы принимают значения $1 / 2$ при $P=1 / 2$. Таким образом, мы видим, что формула «Ян завтра в полдень будет дома или Ян завтра в полдень не будет дома» не является истинной, что противоречит исходным посылкам введения третьего истинностного значения.

Поскольку добавление третьего истинностного значения было достаточно формальным и не привело к ожидаемой семантической интерпретации, то Я. Лукасевич, естественно, перешел к многозначным логикам, которые являются обобщениями его трехзначной. Обозначив третье истинностное значение как $1 / 2$ и руководствуясь интуицией линейного времени, Лукасевич стал принимать в качестве и других значений дробные величины. В отличие от Васильева, у которого истинностные значения качественно различны и линейно не упорядочены. Таким образом, в $n$-значной логике Лукасевича множество истинностных значений принадлежит единичному отрезку $-0,1 /(n-1), 2 /(n-1)$, $\ldots(n-2) /(n-1), 1$.

Волюнтаризм в построении истинностных таблиц в трехзначной логике Лукасевича может приводить к появлению других логических 
систем, в которых берутся другие возможные варианты определения логических связок. Это, например, трехзначная интуиционистская логика А. Гейтинга [22], логика Поста [27] и др. Но получены были эти логики из своих исходных посылок и имеют свои семантические интерпретации.

Совершенно особняком стоит трехзначная логика Д.А. Бочвара $B_{3}$ [1], во многом перекликающаяся с идеями Н.А. Васильева. Бочвар предложил эту логику для анализа логических и семантических парадоксов. Он интерпретировал третье истинностное значение как «бессмысленное» и, как и Васильев, не предполагал его промежуточности между истиной и ложью. Так же, как и Н.А. Васильев, Д.А. Бочвар рассматривает в логике два уровня - внешний, металогический, уровень метаязыка, на котором выполняются законы классической логики, и внутренний, неклассический. В связках внешнего уровня бессмысленное истинностное значение отождествляется с 0 (false), а на внутреннем уровне отрицание берется как у Лукасевича (но в терминах Бочвара отрицание бессмысленного снова бессмысленно), и таблицы истинности для остальных связок тоже являются следствием интерпретации третьего истинностного значения как бессмысленного - хотя бы одного бессмысленного аргумента оказывается достаточно для бессмысленности всего выражения. Несмотря на то что Д.А. Бочвар не предполагал линейной упорядоченности множества истинностных значений, обычно его логику рассматривают с линейной шкалой и многозначные обобщения его логики предполагают именно линейную шкалу [8].

Пытаясь преодолеть семантические противоречия своей логики, Лукасевич создал ее модальные варианты, один из которых имеет нелинейную шкалу истинностных значений.

В 1930 г. Лукасевич предложил модальный вариант трехзначной логики [24], в котором поставил задачу дать такое определение оператора возможности, что все модальные предложения логики Аристотеля имели бы в ней интерпретацию. Такое определение оператора возможности М $P$ имеет вид: $\mathrm{M} P=\sim P \rightarrow P$, что означает «возможно, что $P \gg$ или «если не- $P$, то $P$ ». Оператор необходимости $\mathrm{L} P$, следовательно, имеет вид: $\mathrm{L} P=\sim \mathrm{M} \sim P$. Очень интересен также оператор случайности или неопределенности $\mathrm{Q} P: \mathrm{Q} P=\mathrm{M} P \wedge \mathrm{M} \sim P$, который выделяет третье истинностное значение: $\mathrm{Q}^{1 / 2}=1 ; \mathrm{Q} 1=\mathrm{Q} 0=0$. Смысл этого оператора в том, что описывая будущие случайные события как возможные, с истинностным значением $1 / 2$, мы получаем истинность высказывания «возможно, что Ян завтра в полдень будет дома и, возможно, что Ян 
завтра в полдень не будет дома», что не получалось без использования операторов модальности. Использование оператора случайности также позволяет получить закон исключенного 4-го Васильева: $P \vee \sim P \vee$ $\mathrm{Q} P$ и закон паранепротиворечия в форме: $\sim(P \wedge \sim P \wedge \sim \mathrm{Q} P)$. Это закон исключенного 4-го для силлогистики Аристотеля, который Н.А. Васильев получил из анализа частных суждений, а не закон его воображаемой логики, где третий вид суждений является качественно отличным от утвердительного и отрицательного и составляет вместе с ними образующие дистрибутивной решетки (§§ 3.2, 3.3).

Тем не менее, при интерпретации логики Лукасевича, как претендующей на описание индетерминизма будущих случайных событий, возникают серьезные затруднения. Проблема в том, что определения Лукасевича $1 / 2=\sim 1 / 2,1 / 2=1 / 2 \wedge 1 / 2,1 / 2=1 / 2 \vee 1 / 2$ противоречат нашей интуиции, которая оценивает истинность таких выражений независимо от того, относится высказывание к будущему или нет, и поэтому с помощью таких определений не удается дать адекватную интерпретацию [26]. Впоследствии Я. Лукасевич, не удовлетворенный несоответствием своей логики исходным посылкам, предложил 4-значную $L$ модальную логику [11], в которой увеличивает число модальных операторов. Эта логика уже имеет простейшую нелинейную решетку в качестве шкалы истинностных значений, наподобие логики Васильева. Множество истинностных значений получается прямым произведением множества истинностных значений классической логики на себя (рис. 3). Получается дистрибутивная решетка четырех истинностных значений, где ее образующие «u»и «d»- два значения, соответствующих «возможности».

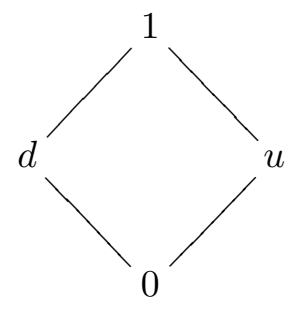

Рис. 3

Логические связки определяются как решеточные операции. Эта логика хоть и многозначная, но классическая, поэтому в ней выполняются законы исключенного третьего и непротиворечивости. Но поскольку теперь есть два варианта обозначения возможности, то Лукасевич 
определяет два оператора возможности и по два оператора необходимости и случайности. Однако были обнаружены совершенно интуитивно неприемлемые формулы, которые опровергают интерпретацию введенных модальных операторов как «возможность» и «необходимость» [7].

А.Р. Андерсон и Н.Д. Белнап [19] предложили рассматривать только такие формулы, в которые импликация входит только один раз и разделяет формулу на антецедент и консеквент, и получили эту же решетку. Все связки у них также решеточные, за исключением отрицания, которое имеет вид: $\sim u=u, \sim d=d$, а для 0 и 1 как обычно. Существуют различные расширения логики Белнапа, в том числе и с другой импликацией [8].

\section{5. Нечеткая логика}

Все рассмотренные раньше формальные логические многозначные системы естественно выражаются на языке нечеткой логики. Но нечеткая логика имеет обобщение, которое еще не было представлено. Нечеткая логика основана на теории нечетких множеств и позволяет давать неточные истинностные оценки. Она предназначена для анализа систем, в которых имеют место человеческие рассуждения и неопределенные понятия. Эта теория началась со статьи Л. Задэ в 1965 г. [29] и с тех пор бурно развивается. В этой теории следует различать два уровня нечеткости. Первый связан с понятием степени принадлежности $\mu_{A}(x)$ элемента $x$ множеству $A$. Степень принадлежности выражается характеристической функцией со значениями в некоторой решетке $M$ :

$$
\mu_{A}(x)= \begin{cases}1, & \text { наибольшая степень принадлежности, } x \in A \\ \nu, & \text { промежуточные степени принадлежности, } \\ & \nu \in M, x \in \in_{\nu} A \\ 0, & \text { наименьшая степень принадлежности, } x \notin A\end{cases}
$$

Соответственно нечеткое подмножество - это подмножество элементов $A$, которые имеют одну степень принадлежности. Такие шкалы используют для оценки истинности выражений типа «небольшой», «не очень большой» и т.п. В логике Н.А. Васильева, 4-значной логике Я. Лукасевича и в логике Белнапа шкалы истинностных значений являются дистрибутивными решетками и степени принадлежности соответствуют степеням истинности. Но обычно в качестве шкалы $M$ принимают отрезок $[0,1]$. Если $\mathrm{M}=0,1$, то нечеткие подмножества становятся обычными, четкими. 
На решетке $M$ обычным образом определяются решеточные операции, которые являются интерпретациями логических операций. В этом случае такая нечеткая логика становится обычной многозначной логикой в духе Васильева (§ 3.3). Но если шкала линейна, т.е. $M=[0,1]$, то отрицание определяется как в большинстве линейных шкал: $\sim \nu=1-\nu$. Тем не менее, такая нечеткая логика не является логикой Лукасевича, поскольку в ней не определяется импликация Лукасевича.

Второй уровень нечеткости, который и представляет дальнейшее обобщение шкалы истинностных значений, связан с тем, что оценкой истинности может быть не только элемент множества $M$, но и его, опятьтаки нечеткое, подмножество. Это так называемая нечеткозначная логика [28]. В ней к нечетким относится само понятие степени истинности. Так, например, если высказывание « $X$ является высоким человеком» имеет степень истинности 0,25 в нечеткой, т.е. многозначной логике, то в нечеткозначной само это значений 0,25 принадлежит нечеткому множеству «относительно невысоких людей». Это легко проиллюстрировать в категорных терминах аналогично § 3.3:

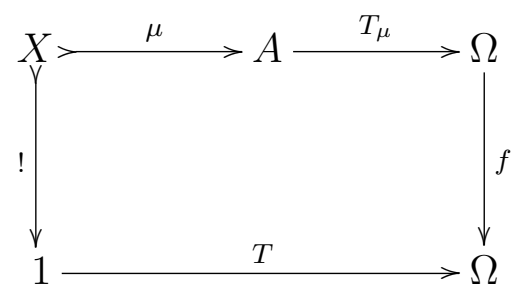

Рис. 4

На этом рисунке характеристическая функция $\mathrm{T}_{\mu}$ подобъекта $\mu$ в общем случае является не элементом объекта истинностных значений $\Omega$, а его подобъектом, и становится элементом только в случае, когда характеристическая функция композиции подобъекта $\mu$ и его характеристической функции тождественна: $f=i d$.

Нечеткозначная логика является основой приближенных рассуждений, в которых используются неопределимые точно или количественно понятия. Для работы в такой ситуации недостаточно просто многозначных логик потому, что кроме неточной оценки истинности терма нужно еще так же неточно оценивать истинность вывода. Таким образом, в нечеткозначной логике являются неточными и значения истинности, и правила вывода. Однозначного определения таких правил 
вывода пока нет. Но это не все трудности - для нечетких подмножеств с нечеткой характеристической функцией иначе надо определять решеточные операции. Возможно, что категорная интерпретация нечеткой логики поможет прояснить эту ситуацию.

\section{6. Заключение}

Мы рассмотрели в этой работе историю понятия многозначности в логике. Мы видели, что уже в Древней Индии логическая многозначность возникла, по сути, как множественность форм отрицания. Позднее, уже в XX веке, Н.А. Васильев на основе возможной вариативности понимания логики Аристотеля приходит к идее многозначности в результате глубокого осмысления понятия отрицания. И мы видели, что частный случай его логики почти дословно совпадает с одной из форм логики Древней Индии.

Я. Лукасевич проблемой отрицания не занимался, он строил свою систему символической логики исходя из других мотивов. Но все логики с такой же, как у Лукасевича, шкалой истинностных значений различаются, в первую очередь, по своему определению отрицания. И логики с нелинейными шкалами тоже классифицируются по этому признаку отрицание может быть решеточным, как в интуиционистской логике, или в приведенной модели логики Васильева, или в $L$-модальной логике Лукасевича, или искусственным, как в логике Белнапа.

Таким образом, первый вывод состоит в том, что различные варианты многозначности в логике порождаются, в основном, трактовкой операции отрицания независимо от исходных мотивов их создателей. Но важны также и интерпретации самих истинностных значений и операций - семантика формальных систем.

И здесь мы приходим ко второму выводу - использование для решения семантических проблем внутри теории внешних средств не приводит к желаемому результату. Каждая система имеет ту содержательную семантику, которая порождается ее внутренней структурой, и привлечение иных структур порождает и иную семантику.

Действительно, успех Н.А. Васильева был обусловлен тем, что он работал внутри силлогистики Аристотеля, использовал ее внутренние средства и провел анализ ее проблем, развивая, по сути, представления Аристотеля. Напротив, Я. Лукасевич, обозначив проблему в логике Аристотеля, формальным образом привлек для ее решения внешние средства символической логики без глубокого содержательного анализа внутри самой этой системы, его определения отрицания и импликации 
произвольны и семантически не обоснованы. Не удивительно, что полученная система, которая оказалась очень интересной, тем не менее, не имеет отношения к той проблеме, решить которую она была призвана.

В отличие от Лукасевича, Д.А. Бочвару удалось построить формальную систему на основе единой согласованной интерпретации третьего истинностного значения во всех связках (любая операция с бессмысленностью снова бессмысленна) и его система имеет содержательное обоснование, как и логика Васильева.

Вообще, категорная интерпретация логики Н.А. Васильева оказала существенную помощь в понимании его идей. Удалось придать естественный смысл его индифферентному высказыванию путем определения отрицания «в некотором смысле», прояснить соответствие понимания Васильевым различных типов суждений образующим дистрибутивной решетки и его представления об отрицании решеточному отрицанию. Выяснилось, что закон паранепротиворечия по Васильеву можно интерпретировать как конъюнкцию утверждения и его отрицания «в некотором смысле» и что выполняется он в определенных топосах с небулевой решеткой истинностных значений, что несколько корректирует выводы Н.А. Васильева о качественно различных типах суждений (образующих решетки) в воображаемой логике - по крайней мере некоторые из них должны иметь пересекающиеся денотаты для того, чтобы логика была неклассической.

\section{Литература}

[1] Бочвар Д.А. Об одном трехзначном исчислении и его применении к анализу парадоксов классического расширенного функционального исчисления // Математический сборник. 1938. Т.4. № 2. С. 287-308.

[2] Васильев Н.А. Логика и металогика // Васильев Н.А. Воображаемая логика. Избранные труды. М.: Наука, 1989. С. 94-123

[3] Васильев Н.А. О частных суждениях, о треугольнике противоположностей, о законе исключенного четвертого // Васильев Н.А. Воображаемая логика. Избранные труды. М.: Наука, 1989, С. 12-53

[4] Васильев Н.А. Воображаемая (неаристотелева) логика // Васильев Н.A. Воображаемая логика. Избранные труды. М.: Наука, 1989, С. 53-94.

[5] Древнеиндийская философия. М.: Мысль, 1972. 363 с.

[6] Зайцев Д.В., Маркин В.И. Воображаемая логика-2: реконструкция одного из вариантов знаменитой логической системы Н.А. Васильева // Труды научно-исследовательского семинара логического центра Института философии РАН 1998. М.: ИФ РАН, 1999. С. 134-142. 
[7] Ивин А.А. Парадоксы модальной логики Я. Лукасевича // Философские науки. 1980, № 1, С. 75-83.

[8] Карпенко А.C. Развитие многозначной логики. М.: Изд. ЛКИ, 2010. 444 c.

[9] Костюк Т.П., Маркин В.И. Формальная реконструкция воображаемой логики Н.А. Васильева // Современная логика: проблемы теории, истории и применения в науке. Материалы V Общероссийской научной конференции. СПб.: Изд. СПбГУ, 1998. С. 154-159.

[10] Легович Ю.С. Максимов Д.Ю. Логические модели выбора решения в самоорганизующихся системах // Проблемы управления. 2013. № 3. C. $18-26$.

[11] Лукасевич Я. Аристотелевская силлогистика с точки зрения современной формальной логики. М.: ИЛ, 1959. 313 с.

[12] Лукасевич Я. О детерминизме // Логические исследования. 1993. Вып. 2. C. $190-205$.

[13] Маковельский А.О. История логики. М.: Наука, 1967. 502 с.

[14] Максимов Д.Ю. Реконфигурирование системной иерархии методами многозначной логики // Автоматика и телемеханика. 2016. № 3. С. 123136.

[15] Маркин В.И. Погружение воображаемой логики Н.А. Васильева в кванторную трехзначную логику // Логические исследования. 2000. Вып. 7. C. $252-260$.

[16] Смирнов В.А. Логические идеи Н.А. Васильева и современная логика // Василъев Н.A. Воображаемая логика. Избранные труды. М.: Наука, 1989. C. 229-260.

[17] Теренътев A.A. K интерпретации логико-методологических схем индийской религиозной философии. // Философские вопросы буддизма. Н.: Наука, 1984. С. 59-72.

[18] Чаттерджи С., Датта Д. Древняя индийская философия. М.: Изд. иностр. лит., 1954. 408 с.

[19] Anderson A.R., Belnap N.D. The pure calculus of entailement // The Journal of Simbolic Logic. 1962. Vol. 27(1). P. 19-52.

[20] Arruda A.J. On the imaginary logic of N.A. Vasiliev // Proceedings of Fourth Latin-American Symposium of Mathematical Logic / Eds. by A.I.Arruda, R.Chuaqui and N.C.A. da Costa. North-Holland, 1979. P. 1-41.

[21] Basham A.L. The History and Doctrines of Ajivikas. London: Luzac, 1951. 316 p.

[22] Heiting A. Die Formalen Regeln der intuitionistischen Logic // Sitzungsberichte der Preussischen Academie der Wissenschaften. Berlin: Phys.-Math. Klasse, 1930. P. 42-56. 
[23] Lucasiewicz J. On three-valued logic // Lucasiewicz J. Selected works / Ed. by L. Borkowski. Amsterdam: N.-H. Pub.Co., 1970. P. 87-88.

[24] Lucasiewicz J. Philosophical remarks on many-valued systems of propositional logic // Lucasiewicz J. Selected works / Ed. by L. Borkowski. Amsterdam: N.-H. Pub.Co., 1970. P. 153-178.

[25] Maximov D.Yu. N.Vasiliev's logic ideas and categorical semantic of ManyValued Logic // Logica Universalis. 2016. Vol. 10. P. 1-23.

[26] Mox Shaw-Kwei Logical paradoxes for many-valued system // The Journal of Symbolic Logic. 1954. Vol. 19(10). P. 37-40.

[27] Post E.L. Introduction to a general theory of elementary propositions // From Frege to Gedel: A Source Book in Mathematical Logic, 1879-1931 / Ed. by Jean van Heijenoort. Cambridge: Camb. Univ. Press, 1967. P. 264-283.

[28] Zadeh L.A. The concept of a linguistic variable and its application to approximate reasoning // Information and Control. 1975. № 8. P. 199-249.

[29] Zadeh L.A. Fuzzy sets // Information and Control. 1965. № 8. P. 338-353. 


\author{
D.Yu. MAXIMOV
}

\title{
N.A. Vasiliev's Logic and Many-valued Logics
}

\author{
Maximov Dmitriy Yurievich \\ V. A. Trapeznikov Institute of Control Sciences, Russian Academy of Sciences. \\ 65 Profsoyuznaya St., Moscow, 117997, Russian Federation. \\ E-mail: phoenixjhanjaa@yandex.ru
}

The history of the notion of many-valuedness is considered in unified manner as the history of negation. The main attention is being paid to N.A. Vasiliev logic, its connections with indian logic of syadvada, with Lukasiewicz logics and logic of D. Bochvar. Many-valued nature of Vasiliev logic and it's formal model in topoi are cleared. A new notion of negation "from some viewpoint" is introduced in topoi. Then Vasiliev set of statement types appears as the set of elements forming a truth values distributive lattice of a topos. These elements translates one into the other by negation "from correspondent viewpoint" and Vasiliev negation understanding corresponds to lattice negation (pseudo-compliment). Vasiliev understanding of an excluded $n$-th rule and paraconsistency is discussed. Vasiliev considered his excluded $n$-th rule as disjunction of lattice forming elements, as disjunction of different negated "from some viewpoint" statement construction ways, and consistent rule as conjunction of a statement and its negation "from some viewpoint". The conditions of these rules modeling in categories are specified.

Keywords: Vasiliev logic, many-valued logic, categorical semantics

\section{References}

[1] Bochvar, D.A. "Ob odnom trekhznachnom ischislenii i ego primenenii $\mathrm{k}$ analizu paradoksov klassicheskogo rasshirennogo funktsional'nogo ischisleniya" [On a three-digit year and its application to the analysis of the paradoxes of the classical extended functional calculus], Matematicheskii sbornik [Mathematical Collection]. 1938. T.4. 2. pp. 287-308. (In Russian)

[2] Vasil'ev, N.A. "Logika i metalogika" [Logic and metalogic], in: N.A. Vasil'ev, Voobrazhaemaya logika. Izbrannye trudy [Imaginary Logic. Selected Works]. M.: Nauka, 1989. pp. 94-123. (In Russian)

[3] Vasil'ev, N.A. "O chastnykh suzhdeniyakh, o treugol'nike protivopolozhnostei, o zakone isklyuchennogo chetvertogo" [On the private judgments of the triangle of opposites, of the law of excluded fourth], in: N.A. Vasil'ev, Voobrazhaemaya logika. Izbrannye trudy [Imaginary Logic. Selected Works]. M.: Nauka, 1989, pp. 12-53. (In Russian)

[4] Vasil'ev, N.A. "Voobrazhaemaya (nearistoteleva) logika" [Imaginary (nearistoteleva) logic], in: N.A. Vasil'ev, Voobrazhaemaya logika. Izbrannye trudy [Imaginary Logic. Selected Works]. M.: Nauka, 1989, pp. 53-94. (In Russian) 
[5] Drevneindiiskaya filosofiya [Ayurveda philosophy]. M.: Mysl', 1972. 363 pp. (In Russian)

[6] Zaitsev, D.V., Markin V.I. "Voobrazhaemaya logika-2: rekonstruktsiya odnogo iz variantov znamenitoi logicheskoi sistemy N.A. Vasil'eva" [Imaginary Logic 2: reconstruction of one of the variants of the famous logic systems NA Vasilyeval, Trudy nauchno-issledovatel'skogo seminara logicheskogo tsentra Instituta filosofii RAN 1998 [Proceedings of the Scientific-Research Seminar logical center of the Institute of Philosophy, 1998]. M.: IF RAN, 1999. pp. 134-142. (In Russian)

[7] Ivin, A.A. "Paradoksy modal'noi logiki Ya. Lukasevicha" [Paradoxes of modal logics of Lukasiewicz], Filosofskie nauki [Philosophical Sciences], 1980, 1, pp. 75-83. (In Russian)

[8] Karpenko, A.S. Razvitie mnogoznachnoi logiki [The development of multivalued logic]. M.: Izd. LKI, 2010. 444 pp. (In Russian)

[9] Kostyuk, T.P., Markin, V.I. "Formal'naya rekonstruktsiya voobrazhaemoi logiki N.A. Vasil'eva" [The formal reconstruction of an imaginary logic NA Vasilyeva], Sovremennaya logika: problemy teorii, istorii $i$ primeneniya $v$ nauke. Materialy V Obshcherossiiskoi nauchnoi konferentsii [ Modern logic: problems of the theory, history and application in science. Proceedings of the V All-Russian scientific conference]. SPb.: Izd. SPbGU, 1998. pp. 154-159. (In Russian)

[10] Legovich, Yu.S., Maksimov, D.Yu. "Logicheskie modeli vybora resheniya v samoorganizuyushchikhsya sistemakh" [Logic model selection decisions in self-organizing systems], Problemy upravleniya [Problems of management], 2013, no 3, pp. 18-26. (In Russian)

[11] Lukasevich, Ya. Aristotelevskaya sillogistika s tochki zreniya sovremennoi formal'noi logiki [Aristotelian syllogistic terms of modern formal logic]. M.: IL, 1959. 313 pp. (In Russian)

[12] Lukasevich, Ya. "O determinizme" [About determinism], Logicheskie issledovaniya [Logical Investigations], 1993, vol.2, pp.190-205. (In Russian)

[13] Makovel'skii, A.O. Istoriya logiki [History of logic]. M.: Nauka, 1967. 502 pp. (In Russian)

[14] Maksimov, D.Yu. "Rekonfigurirovanie sistemnoi ierarkhii metodami mnogoznachnoi logiki" [Reconfiguring the system hierarchy of methods of multi-valued logic], Avtomatika i telemekhanika [Automation and Remote Control], 2016, № 3, pp. 123-136. (In Russian)

[15] Markin, V.I. "Pogruzhenie voobrazhaemoi logiki N.A. Vasil'eva v kvantornuyu trekhznachnuyu logiku" [Dive imaginary logic NA Vasilyeva quantifier in three-valued logic], Logicheskie issledovaniya [Logical Investigations], 2000, vol.7, pp. 252-260.(In Russian)

[16] Smirnov, V.A. "Logicheskie idei N.A. Vasil'eva i sovremennaya logika" [The logical idea NA Vasilyev and modern logic], in: N.A. Vasil'ev, 
Voobrazhaemaya logika. Izbrannye trudy [Imaginary logic. Selected works]. M.: Nauka, 1989, pp. 229-260. (In Russian)

[17] Teren'tev, A.A. "K interpretatsii logiko-metodologicheskikh skhem indiiskoi religioznoi filosofii" [The interpretation of the logical and methodological schemes of the Indian religious philosophy], Filosofskie voprosy buddizma [Philosophical Problems of Buddhism]. N.: Nauka, 1984, pp. 59-72. (In Russian)

[18] Chatterdzhi, S., Datta, D. Drevnyaya indiiskaya filosofiya [Ancient Indian philosophy]. M.: Izd. inostr. lit., 1954. 408 pp. (In Russian)

[19] Anderson, A.R., Belnap, N.D. "The pure calculus of entailement", The Journal of Simbolic Logic, 1962, vol. 27(1), pp. 19-52.

[20] Arruda, A.J. "On the imaginary logic of N.A. Vasiliev", Proceedings of Fourth Latin-American Symposium of Mathematical Logic, eds. by A.I. Arruda, R. Chuaqui and N.C.A. da Costa. North-Holland, 1979. pp. 1-41.

[21] Basham, A.L. The History and Doctrines of Ajivikas. London: Luzac, 1951. $316 \mathrm{pp}$.

[22] Heiting, A. "Die Formalen Regeln der intuitionistischen Logic", Sitzungsberichte der Preussischen Academie der Wissenschaften, Berlin: Phys.-Math. Klasse, 1930. pp. 42-56.

[23] Lucasiewicz, J. "On three-valued logic", in: J Lucasiewicz, Selected works, ed. by L. Borkowski. Amsterdam: N.- H. Pub.Co., 1970, pp. 87-88.

[24] Lucasiewicz, J. "Philosophical remarks on many-valued systems of propositional logic", in: J Lucasiewicz Selected works, ed. by L. Borkowski. Amsterdam: N.- H. Pub.Co., 1970, pp. 153-178.

[25] Maximov, D.Yu. "N.Vasiliev's logic ideas and categorical semantic of manyvalued logic", in: J Lucasiewicz, Logica Universalis, ed. by L. Borkowski. 2016, vol. 10, pp. 1-23.

[26] Mox, Shaw-Kwei. "Logical paradoxes for many-valued system", The Journal of Symbolic Logic, 1954, vol. 19(10), pp. 37-40.

[27] Post, E.L. "Introduction to a general theory of elementary propositions", From Frege to Gedel: A Source Book in Mathematical Logic, 1879-1931, ed. by Jean van Heijenoort. Cambridge: Camb. Univ. Press, 1967. pp. 264-283.

[28] Zadeh, L.A. "The concept of a linguistic variable and its application to approximate reasoning", Information and Control, 1975, no 8, pp. 199-249.

[29] Zadeh, L.A. "Fuzzy sets", Information and Control, 1965, no 8, pp. 338-353. 